resources in an online PN "toolkit" available to support people diagnosed with STI is ideal.

Disclosure of interest statement The authors have no conflicts of interest to disclose.

\section{P02.05 SEXUAL HEALTH LITERACY - AN EMERGING FRAMEWORK FOR RESEARCH AND INTERVENTION TO IMPROVE SEXUAL HEALTH FOR GAY MEN}

${ }^{1,2,3} \mathrm{M}$ Gilbert ${ }^{*},{ }^{4,5} \mathrm{O}$ Ferlatte, ${ }^{3} \mathrm{~W}$ Michelow, ${ }^{6} \mathrm{~S}$ Martin, ${ }^{6}$ Y Young, ${ }^{7} \mathrm{~L}$ Donelle, ${ }^{8} \mid$ Rootman, ${ }^{6} \mathrm{~L}$ McDaid, ${ }^{9} \mathrm{P}$ Flowers. ${ }^{1} B C$ Centre for Disease Control; ${ }^{2}$ Ontario HIV Treatment Network; ${ }^{3}$ University of British Columbia; ${ }^{4}$ Simon Fraser University; ${ }^{5}$ Community-Based Research Centre for Gay Men's Health; ${ }^{6}$ University of Glasgow; ${ }^{7}$ Western University; ${ }^{8}$ University of Victoria; ${ }^{9}$ Glasgow Caledonian University

10.1136/sextrans-2015-052270.226

Introduction Health literacy is an important determinant of health yet has not been formally applied to sexual health. An expert event brought 38 researchers, community members, and service providers together to explore gay men's sexual health literacy (SHL).

Methods Using a World Café method, three rounds of discussion posed questions about SHL in relation to gay men, providers, and underlying systems. Documented notes were analysed thematically through two rounds of group synthesis and a subsequent review by one investigator.

Results SHL was influenced by ways men access information, through peer networks, and coded communications with prospective partners. The Internet influences access to, delivery of and engagement with information, while new technologies and changing sexual norms complicate message consistency and risk assessment. Actionable risk assessment requires numeracy skills. Gay men were generally perceived to have high SHL with concern that SHL may be lower for some sub-populations (e.g., youth, immigrant men). Participants valued bottom-up/community-based over top-down/expert models of providing SHL. Health care provider attitudes, cultural competency, knowledge and communication skills were seen as key determinants of SHL and non-traditional health sectors also play key roles (e.g., education, pornography). Underlying determinants of SHL included stigma related to HIV or sexual orientation, access and organisation of health care services, systemic shifts to self-care models, political ideologies and funding. Syndemic, intersectional, and holistic health approaches to SHL were advocated. Participants recognised the strong history of activism and mobilisation within gay communities as integral to SHL.

Conclusion Critical health literacy has been a core component of the gay men's health movement to date. Innovations in biomedical technologies and access to online information are critically shaping experiences of SHL for gay men. The expanded theoretical framework emerging from these findings serves as a starting point to inform enhancing SHL and designing effective interventions.

Disclosure of interest statement The authors have no conflict of interest to disclose.

\section{P02.06 IDENTITIES IN MOTION: CYBERSPACE AND MYANMAR MEN HAVING SEX WITH MEN}

Lin Khine Soe*, Putten Marc Van der*. Faculty of Public Health, Thammasat University, Rangsit Campus, Klong Luang Piyachart Bld. 10th Fl. Pathumthani, 12121, Thailand

10.1136/sextrans-2015-052270.227
The enquiry was financed by the World Society Foundations and the Thabyay Education Network.

This paper explores how Myanmar men-having-sex-with-men (MSM) create identity through lived and mediated realities, by using cyber social networks, 30 in-depth interviews were conducted through snowballing among MSM cyber social network members. Content analysis was guided by a social network analysis framework. Networking positively affected connectedness and social support however norms and values were challenged. Age, experience, sex-roles, and peer-relationships seem to influence self-efficacy. Interpersonal dynamics were driven by onlinecruising, dating, cyber-sex and off-line encounters. In conclusion, access to social networking offers an outlet for socio-cultural and legal oppressed Myanmar MSM, however building life-skills among younger users' needs attention. Social networks could be appropriate channels for social and health programs targeting MSM.

\section{P02.07 UTILISATION OF RISK SCORE TOOL OF INTERNET I WANT THE KIT (IWTK) HOME SELF-COLLECTION PROGRAM FOR SEXUALLY TRANSMITTED INFECTIONS (STIS) IN MALES}

${ }^{1} \mathrm{Y}$-H Hsieh*, ${ }^{2} \mathrm{M}$ Jett-Goheen, ${ }^{2} \mathrm{~L}$ Dize, ${ }^{2} \mathrm{M}$ Barnes, ${ }^{2} \mathrm{P}$ Barnes, ${ }^{1,2} \mathrm{CA}$ Gaydos. ${ }^{1}$ Department of Emergency Medicine; 'Infectious Diseases, Johns Hopkins University, Baltimore, Maryland, USA

\subsection{6/sextrans-2015-052270.228}

Introduction In our previous pilot study on the internet I Want The Kit (IWTK) home self-collection program for sexually transmitted infections (STIs), a voluntary risk score tool predicted STIs well for the female volunteers but not for the males. We investigated the association of IWTK risk score and the presence of STIs in male users when the risk score quiz became mandatory for the program.

Methods A six-question quiz which includes demographic and sexual risk behaviour became a mandatory part of IWTK in August 2103. This analysis was restricted to male participants living in Maryland and Washington DC using IWTK August 2013-April 2015. Cochran-Armitage trend test was performed to determine if the prevalence of STIs (chlamydia, gonorrhoea, or trichomonas infection) increased with the higher score of risk score category.

Results Overall, 592 male participants submitted specimens for STI testing and completed risk score quizzes. The majority $(57 \%)$ were $<30$ years (mean: $30.1 \pm 9.3$ years); $42 \%$ white, $42 \%$ black, and $16 \%$ other races. $203(34.3 \%)$ resided in zip codes of Baltimore City. The majority $(53 \%)$ of the participants had risk score of 4-6, followed by scores of $0-3(25 \%)$, and 7-10 (22\%). The overall prevalence of STIs was 10.5\% (62/ 592). The prevalence of STIs was $6.2 \%$ for users with risk score of $0-3,10.9 \%$ for those with $4-6$, and $14.3 \%$ for those with 7-10 (trend test: $p=0.026)$. 52\% (13/25) of STI positive participants resided in three zip codes located in central and northeast Baltimore City and participants in these three zip codes had a marginally higher risk score than others $(p=0.082)$.

Conclusions After excluding potential selection bias, IWTK risk score tool predicted the presence of STIs for male users and could potentially be used for identifying hotspots for STI intervention.

Disclosure of interest statement The research group has received research funding from U54EB007958, NIBIB, NIH; AI06861301, NIH, NIAID. 